\title{
Ab Initio Simulations of Liquid Carbon Monoxide at High Pressure
}

\author{
Tanis C. Leonhardi ${ }^{\mathrm{a}}$ and Burkhard Militzer ${ }^{\mathrm{a}, \mathrm{b}}$ \\ ${ }^{a}$ Department of Earth and Planetary Science, University of California, Berkeley, CA 94720, \\ USA. \\ ${ }^{\mathrm{b}}$ Department of Astronomy, University of California, Berkeley, CA 94720, USA. \\ Corresponding Author: Tanis C. Leonhardi \\ e-mail address: tcleonhardi@berkeley.edu \\ Postal Address: \\ Department of Earth and Planetary Science \\ University of California, Berkeley \\ 307 McCone Hall \\ Berkeley, CA 94720-4767
}

\begin{abstract} interiors, and as a plasma at high-T.

\section{Keywords}

- carbon monoxide

- density functional theory

- first principles molecular dynamics

- equation of state

- planetary interiors
\end{abstract}

Carbon monoxide occurs as a volatile species in the interiors of terrestrial planets, and as a disequilibrium atmospheric constituent in the giant planets. It plays an important role during the accretionary stages of planet formation reacting with gases to form compounds such as $\mathrm{CH}_{4}$ and $\mathrm{H}_{2} \mathrm{O}$. The structure of carbon monoxide is unknown over the majority of the temperature and pressure regime in giant planet interiors. Here we perform ab initio molecular dynamics simulations to characterize $\mathrm{CO}$ to $140 \mathrm{GPa}$ and 5,000 K. We find that $\mathrm{CO}$ is stable as a molecular liquid at lower P-T conditions, as a polymeric liquid at higher P-T conditions found in ice giant

\section{Introduction}

Carbon monoxide ice is one of the solid phases accreted during the formation of ice giant planets alongside other organic $(\mathrm{C}, \mathrm{N}, \mathrm{O}, \mathrm{H})$-rich ices and dust grains $[1,2,3]$. These ice particles react with gases and other accreted materials present during, and after, planetary accretion to form compounds, including $\mathrm{CH}_{4}$ and $\mathrm{H}_{2} \mathrm{O}$, which are commonly observed in giant planet atmospheres today $[1,2,3,4,5,6]$. CO ice is a phase commonly invoked as one of the ices accreted during planetary formation $[1,2,3]$. A recent study developed a 'volatile distribution model' in which chemical and physical dynamics of the protoplanetary disk are incorporated with an accretionary planet formation model for Uranus and Neptune to explain trace elemental and isotopic

\section{Page 1 of 9}


differences observed today between these two planets [3]. Thus understanding the structure and behavior of $\mathrm{CO}$ at the temperatures and pressures of giant planet interiors is important for understanding the physical conditions during the first stages of giant planet formation and evolution.

Determining the structure, stability, and properties of planetary ices composed of C-H-O-N compounds at high temperatures and pressures can help to understand magnetic field generation in ice giant planets. One way proposed to generate the magnetic fields in Uranus and Neptune is through convection in layers of ices with low conductivity $[7,8,9,10,11,12]$. It is important to point out that 'ice' refers to the material's state during planet formation, now these materials may exist in liquid or superionic form in the hot interiors of giant planets [13].

Despite the relatively high abundance of $\mathrm{C}$ and $\mathrm{O}$ in the ice giants, only one experimental study has explored the stability and properties of CO at GPa pressure [14]. In the study, Nellis et al. [14] used a combination of experiments and numerical calculations to determine the shock Hugoniot curve of liquid $\mathrm{CO}$ from 5 to $60 \mathrm{GPa}$. This means the structure and properties of $\mathrm{CO}$ remain unknown over the majority of the P-T regime inside Neptune's and Uranus's ice layer (reaching up 7,000 K and $600 \mathrm{GPa}$ ) [15,16]. Determining the structure of CO over P-T conditions inside giant planets is important for improving our understanding of the current structure of ice giants and their formation processes.

In this study we use first-principles molecular dynamics simulations based on density functional theory [17] to construct a P-T phase diagram for liquid CO up to $140 \mathrm{GPa}$ and $5000 \mathrm{~K}$. Results from these simulations were used to provide a first constraint on the melting line of $\mathrm{CO}$ at high temperatures and pressures and to study the structure of liquid $\mathrm{CO}$ at high pressure. We compare our results with previous experimental results and one predicted isentrope for Uranus's ice layer [15].

\section{Computational Methods}

First-principles molecular dynamics (FPMD) simulations based on density functional theory (DFT) [17] were performed using the Vienna ab initio Simulation Package (VASP) [18]. These simulations used the generalized gradient approximation (GGA) with a Perdew-Burke-Ernzerhof exchange-correlation functional [19] and projector augmented wave (PAW) pseudopotentials [20]. The Brillouin zone was sampled using $\Gamma$ point only. An energy cut-off value of $1000 \mathrm{eV}$ was used to represent the electronic orbital in a plane-wave basis as it provided convergence of pressures and energies to less than $1 \%$ uncertainty.

All simulations were performed with periodic boundary conditions and temperatures were kept constant by a Nose-Hoover thermostat [21,22]. Previous FPMD simulations of $\mathrm{CO}_{2}$ showed that results are converged with 32 molecules (96 atoms) in the simulation cell [23,24]. Hence we used a 3x3x3 body centered cubic supercell of 54 CO molecules (108 atoms) to mitigate finite size effects. Simulations were run with a $1.0 \mathrm{fs}$ time step and total simulation time varied between 2 and 12 ps in length depending on the system temperature. At higher temperatures, equilibrium is obtained faster, so shorter simulation times can be performed. 
Simulations systematically sampled a VT ensemble (V=volume, T=Temperature) up to $140 \mathrm{GPa}$ and $5,000 \mathrm{~K}$. An additional simulation with a density of $0.82 \mathrm{~g} / \mathrm{cc}$ was performed at $9,000 \mathrm{~K}$ to evaluate the extent of $\mathrm{CO}$ dissociation into $\mathrm{C}$ and $\mathrm{O}$ atoms and study a $\mathrm{CO}$ plasma. The trajectory for each of these simulations was used to determine the atomic coordination of $\mathrm{C}$ atoms. Here we use the terminology employed in previous studies $[23,24]$ to define the carbon coordination as the number of oxygen and carbon atoms within a specified cut-off distance of a given carbon atom. The cut-off distance used for coordination determination was the first minimum of the C-O pair correlation function, see for example Boates et al. [23].

\section{Results and Discussion}

The CO phases observed from each simulation were mapped onto a high pressure, high temperature phase diagram (Fig.1). Calculated pressures and energies for these simulations are reported in Table 1. For the purpose of defining phase boundaries we used a molecular fraction cutoff of 0.5 . This means that the molecular liquid contained $\geq 50 \% \mathrm{CO}$ molecules. Molecules with C-C bonds were classified as C-C polymers. Visualizations of the observed liquid phases and a partially dissociated phase are provided in Fig. 2.

\subsection{Liquid Phases}

At low temperatures and pressures we observed $\mathrm{CO}$ as a molecular liquid. Abundant $\mathrm{CO}$ molecules produced $\mathrm{CO}$ pair correlation functions with a peak at the characteristic distance of $1.128 \AA$ (see Fig. 3), which corresponds to the C-O triple bond length [25]. As we increased the density, the coordination of carbon atoms increased from 1 to 2 , thus becoming more polymerized. The higher $\mathrm{C}$ coordination corresponded to the development of $\mathrm{CO}_{2}$ molecules and polymers with $\mathrm{C}-\mathrm{C}$ bonds, visualized in Fig. 2B. In our phase diagram, polymeric liquid refers to a liquid consisting of $\mathrm{C}-\mathrm{C}$ polymers and $\mathrm{CO}_{2}$ molecules. These new polymers were observed indirectly as a drop in the peak value of the $\mathrm{C}-\mathrm{O}$ pair correlation function, the development of a C-C peak at $\sim 1.38 \AA$, and a O-O peak at $\sim 2.32 \AA$ (Fig. 3). The O-O distribution peak distance has the same value as the distance between oxygen nuclei bound in a $\mathrm{CO}_{2}$ molecule [26]. Single $\mathrm{C}-\mathrm{C}$ bonds have an interatomic distance of $1.54 \AA$, double $\mathrm{C}-\mathrm{C}$ bonds of $1.34 \AA$, and triple $\mathrm{C}-\mathrm{C}$ triple of $1.2 \AA$ [27]. The observed distance of $\sim 1.38 \AA$ for the $\mathrm{C}-\mathrm{C}$ pair correlation function is characteristic of double $\mathrm{C}-\mathrm{C}$ bonds. However additional work investigating the details of $\mathrm{C}-\mathrm{C}$ bonding would need to be preformed to characterize the nature of $\mathrm{C}-\mathrm{C}$ bonds in the polymers observed in Fig. 2B and Fig. 3B.

In addition to analyzing the peaks in the pair correlation functions, we also performed a cluster analysis for the polymeric simulation ' $\mathrm{B}$ '. Two atoms were considered to be part of the same cluster if they are separated by less than $1.90 \AA$. Based on this simple geometric criterion, we determined the clusters for every configuration and then averaged the results along the trajectory. Zero $\mathrm{O}_{2}$ molecules were found in the entire simulation, which implies the $\mathrm{C}-\mathrm{O}$ bonding is strong and a sufficient number of carbon atoms were present to prevent the formation of $\mathrm{O}_{2}$ molecules. 
Our analysis also revealed that $\mathrm{CO}_{2}, \mathrm{CO}$, and $\mathrm{C}_{2} \mathrm{O}_{3}$ clusters occurred most frequently. However, they contained only $28.4 \%, 6.7 \%$ and $4.6 \%$ of the $\mathrm{C}$ atoms, respectively. The majority of the $\mathrm{C}$ atoms, $57.6 \%$, were stored long $\mathrm{C}-\mathrm{O}$ polymers that contained at least $21 \mathrm{C}$ and $8 \mathrm{O}$ atoms. On average, these polymers consisted of $31.1 \mathrm{C}$ and $16.0 \mathrm{O}$ atoms, which implies that a $\mathrm{C}: \mathrm{O}$ ratio of $2: 1$ is particularly favorable. We can thus write the following approximate polymerization reaction,

$$
3 \mathrm{n} \mathrm{CO} \quad \rightarrow \quad \mathrm{n} \mathrm{CO}_{2}+\left[\mathrm{C}_{2 \mathrm{n}} \mathrm{O}_{\mathrm{n}}\right]_{\text {polymer }}
$$

while keeping in mind that a variety of polymers with $\mathrm{n} \geq 21$ were produced. A small remainder of $2.7 \% \mathrm{C}$ atoms were found to occur in small short-lived clusters with up to $6 \mathrm{C}$ atoms.

The transition from a molecular to a polymeric liquid is associated with a decrease in slope of the $\mathrm{P}-\mathrm{V}$ curve at fixed $\mathrm{T}$ shown in Fig. 4. This change in slope is more distinct when plotted as a $\mathrm{P}^{*} \mathrm{~V}$ vs. $\mathrm{V}$ curve in Fig. 4. Molecular dynamics studies on $\mathrm{N}_{2}$ [28], $\mathrm{H}_{2}$ [29], and $\mathrm{CO}_{2}$ [24] found that a plateau in the P-V equation of state for those systems corresponded to a first order liquidliquid phase transition (LLPT). Boates et al. determined that the $\mathrm{CO}_{2}$ first-order LLPT corresponded to a rapid decrease in the 2-coordination of $\mathrm{C}$ and increase in 3-coordination and the formation of stable $\mathrm{CO}_{3}$ molecules [24]. Beyond the LLPT at much higher temperatures and pressures, carbon gradually transition from 3-coordination to short-lived 4-coordination species. The plateau in the CO P-V curve observed in this study may correspond to a first order LLPT similar to that observed for $\mathrm{CO}_{2}$, where the molecular liquid becomes a polymeric liquid. However, with the results from this study we cannot conclusively state whether there is an LLPT between molecular and polymeric CO. Additional work sampling PT space with a finer grid could provide useful on this matter.

\subsection{CO dissociation}

Only one experimental study has explored the structure of $\mathrm{CO}$ at high temperatures and pressures. This study by Nellis et al. (1981) [14] used gas-gun shock experiments to determine the CO Hugoniot curve up to $60 \mathrm{GPa}$. Their P-V results are plotted alongside our results in Fig. 4. For the low pressure $(\leq 10 \mathrm{GPa})$ experimental data points, our results suggest a shock temperature between $2000 \mathrm{~K}$ and $3000 \mathrm{~K}$, which is in agreements with temperature predictions for these shock pressures which spanned 2,000 to 3,000 K [14]. These experiments reported a rapid change in slope of the $\mathrm{P}-\mathrm{V}$ line above $10 \mathrm{GPa}$ [14]. The high pressure $(\geq 10 \mathrm{GPa})$ data points did not fall along the predicted $\mathrm{CO}$ shock Hugoniot curve and were explained by the presence of high density carbon phases, primarily diamond [14]. Temperatures were predicted at $\sim 7,000 \mathrm{~K}$ for the $30 \mathrm{GPa}$ data point and $>10,000 \mathrm{~K}$ for the $50-60 \mathrm{GPa}$ data points. The P-T space sampled in our study only extended to $5,000 \mathrm{~K}$ at these higher pressures, so we cannot provide insight to the state of the system at the physical conditions experienced at those data points.

The $\mathrm{CO}$ shock measurements were interpreted as representing dissociation of $\mathrm{CO}$ molecules into diamond, \pm graphite, $\mathrm{CO}_{2}$, and $\mathrm{O}_{2}$ above $10 \mathrm{GPa}$, resulting in a change of slope in the $\mathrm{P}-\mathrm{V}$ equation of state [14]. The researchers proposed complete dissolution of $\mathrm{CO}$ into diamond occurs by $30 \mathrm{GPa}$ and 7,000 K [14]. Over the 10-30 GPa pressure range and 3,000-7,000 K CO was believed to dissociate into $\mathrm{CO}_{2}$, diamond, and $\mathrm{O}_{2}$, although these phases were not directly 
observed co-existing. Our simulations only reached 5,000 $\mathrm{K}$ in the $\geq 30 \mathrm{GPa}$ range, so we cannot evaluate the state of the system at the higher pressure experimental data points.

Simulations performed along isotherms up to 3,500 K showed plateaus in the P-V slope (Fig. 4). By 5,000 $\mathrm{K}$ there is no observable drop in pressure along the P-V curve, suggesting that a LLPT does not exist at high-T. Evidence of dissociation in the 4-5,000 K simulations is also observed as broadening of pair correlation function peaks and infilling of distribution minima. If $\mathrm{O}_{2}$ formed in a simulation, as is predicted from experimental studies [14] we would expect to see a peak at $1.208 \AA$ [30] in the O-O pair correlation function, a peak at 1.54 in the C-C distribution for the single $\mathrm{C}-\mathrm{C}$ bond, and a peak at $2.32 \AA$ for the interatomic distance between oxygen atoms bound in $\mathrm{CO}_{2} \AA$ [26]. We observed no such O-O peak, suggesting $\mathrm{O}_{2}$ was not produced in our simulations. The only observed peak in the $\mathrm{O}-\mathrm{O}$ pair correlation function for our simulations was a peak at $2.32 \AA$, from which we conclude $\mathrm{CO}_{2}$ was formed. A peak in the $\mathrm{C}$-C distribution at $1.38 \AA$ was common to many simulations, however this does not correspond to the characteristic length of the single $\mathrm{C}-\mathrm{C}$ bond in diamond. These results suggest that $\mathrm{CO}_{2}$ formed, $\mathrm{O}_{2}$ did not, and $\mathrm{C}$ formed polymers with $\mathrm{C}-\mathrm{C}$ bonds rather than diamond or graphite. However, the increased polymerization of $\mathrm{C}$ to form $\mathrm{CO}_{2}$ molecules was observed in our simulations as was predicted by previous experimental results [14].

We performed one additional simulation with a density of $0.82 \mathrm{~g} / \mathrm{cc}$ at $9,000 \mathrm{~K}$ to see if full $\mathrm{CO}$ dissociation was achieved by $9,000 \mathrm{~K}$. The pair correlation functions for this simulation are plotted in Fig. 3. For complete dissociation we expect complete infilling of minima and broadening of maxima to form one smooth distribution. While infilling and broadening are clearly evident in the 9,000 K simulation, there are still pronounced maxima for the C-O and C-C correlation distributions. Molecules with short-lived ( $<15 \mathrm{fs}) \mathrm{C}-\mathrm{C}$ bonds were observed in these simulations. These results show a similar behavior to $\mathrm{CH}_{4}$ [31] and a bulk $\mathrm{C}: \mathrm{H}: \mathrm{O}: \mathrm{N}$ liquid [32] which also developed molecules with short-lived $\mathrm{C}-\mathrm{C}$ bonds at high temperatures. Our results suggest that complete dissociation of $\mathrm{CO}$ has not occurred at $9,000 \mathrm{~K}$ and low pressures. Additional simulations up to and above $10,000 \mathrm{~K}$ at higher pressures can constrain the conditions necessary for full dissociation of $\mathrm{CO}$.

\subsection{Melting line and implications for giant planet interiors}

We used isothermal single-phase compression simulations to determine the $\mathrm{CO}$ melting line. These simulations may experience supercooling as they are compressed. Our simulations froze into an amorphous structure. We assume an ordered $\mathrm{CO}$ solid may be more stable. We also did not reverse this transformation by expanding our frozen simulations. For these reasons, we expect our proposed melting line to be a lower bound in PT space for the true CO melting line. The first completely frozen simulation was designated melting point for a given isotherm. We plotted the resulting melting line along with an isentrope for the temperatures and pressures inside Uranus's ice layer in Fig. 1. Our findings predict that $\mathrm{CO}$ would exist as a polymeric liquid in Uranus' ice layer. Numerical models of magnetic field generation field on Uranus and Neptune compared with observational information about their magnetic favor dynamo generation in a layer of convecting liquid characterized by poor conductivity $[10,11,12]$. Additionally, recent findings have shown that the development of $\mathrm{C}-\mathrm{C}$ bonds in $\mathrm{H}-\mathrm{O}-\mathrm{C}-\mathrm{N}$ liquids greatly affects their conductivity and viscosity, which in turn impacts the magnetic Reynolds number 
and the nature of a magnetic field generated by the liquid [32]. The development of $\mathrm{C}-\mathrm{C}$ in our simulations at high pressures could potentially correlate to changes in $\mathrm{CO}$ properties. Additional work determining the conductivities and transport properties of $\mathrm{CO}$ across the full $\mathrm{P}-\mathrm{T}$ regime inside these planets would be useful in determining if $\mathrm{CO}$ may be an ice contributing to magnetic field generation.

\section{Conclusions and Implications}

The phase diagram constructed in this study is the first P-T phase diagram for $\mathrm{CO}$ extending to $140 \mathrm{GPa}$ and 5,000 K. We found that carbon existed in 1-coordination in CO molecules as a molecular liquid at low temperatures and pressures. Between 8-13 GPa we observed a transition from a molecular liquid to a polymeric liquid. Additional work constraining the conditions of this transition will allow for determination of whether it is a gradual change or an abrupt change that corresponds to a first order liquid-liquid phase transition. Our proposed phase diagram suggests that CO may exist as a polymeric liquid in Uranus's ice layer.

\section{Acknowledgements}

This work was supported in part by NSF and by the U.S. Department of Energy (DOE) grants DE-SC0010517 and DE-SC0016248. Computational resources were provided by the National Energy Research Scientific Computing Center (NERSC).

\section{References}

1. R.G. Prinn, B. Fegley Jr., Kinetic inhibition of $\mathrm{CO}$ and $\mathrm{N}_{2}$ reduction in circumplanetary nebulae: Implications for satellite composition, The Astrophysical Journal 249 (1981) 308-317.

2. G.P. Horedt and W.B. Hubbard, Two and three layer models of Uranus, Moon and the Planets 29 (3) (1983) 229-236.

3. M. Ali-Dib, O. Mousis, J-M. Petit, J.I. Lunine, The measured compositions of Uranus and Neptune from their formation on the CO ice line, The Astrophysical Journal 793 (2014) 9.

4. A. Marten, D. Gautier, T. Owen, D.B. Sanders, H.E. Matthews, S.K. Atreya, First observations of $\mathrm{CO}$ and $\mathrm{HCN}$ on Neptune and Uranus at millimeter wavelengths and the implications for atmospheric chemistry, The Astrophysical Journal 406 (1993) 285-297.

5. K. Lodders, and B. Fegley Jr., Atmospheric Chemistry in Giant Planets, Brown Dwarfs, and Low-Mass Dwarf Stars, Icarus 155 (2) (2012) 393-424.

6. T. Encrenaz, E. Lellouch, P. Drossart, H. Feuchtgruber, G.S. Orton, S.K. Atreya, The detection of carbon monoxide in the atmosphere of Uranus, EOS 115 (35-36) (2004) 3536.

7. W.J. Nellis, D.C. Hamilton, N.C. Holmes, H.B. Radousky, F.H. Ree, A.C. Mitchell, M. Nicol, The nature of the interior of Uranus based on studies of planetary ices at high dynamic pressure, Science 240 (4853) (1988) 779-781. 
8. J.E.P. Connerey, M.H. Acuña, N.F. Hess, The Magnetic Field of Neptune. Journal of Geophysical Research 96 (1991) 19023-19042.

9. J.E.P Connerey, Magnetic Fields of the Outer Planets, Journal of Geophysical Research 98 (E10) (1993) 18659-18679.

10. S. Stanley, J. Bloxham, Convective-region geometry as the cause of Uranus' and Neptune's unusual magnetic fields, Nature 428 (151-153) (2004)151-153.

11. S. Stanley, J. Bloxham, Numerical dynamo models of Uranus' and Neptune's magnetic fields, Icarus 184 (2) (2006) 556-572.

12. S. Stanley, G.A. Glatzmaier, Dynamo models for planets other than earth. Space Sci. Rev. 1 (2009) 1.

13. H.F. Wilson, M.L. Wong, B. Militzer, Superionic phase change in water: consequences for the interiors of Uranus and Neptune, Phys. Rev. Lett. 110 (2013) 151102.

14. W.J. Nellis, F.H. Ree, M. van Thiel, and A.C. Mitchell, Shock compression of liquid carbon monoxide and methane to 90 GPa (900 kbar), J. Chem Phys. 75 (6) (1988) 3055 3063.

15. M. Ross, The ice layer in Uranus and Neptune-diamonds in the sky?, Nature 292 (435436) (1981) 435-436.

16. N. Nettelmann, R. Helled, J.J. Fortney, R. Redmer, New indication for a dichotomy in the interior structure of Uranus and Neptune from the application of modified shape and rotation, Planetary and Space Science 77 (2013) 143-151.

17. P. Hohenberg and W. Kohn, Inhomogeneous Electron Gas, Phys. Rev. 136 (3B) (1964) B864.

18. G. Kress and J. Furthmüller, Efficient iterative schemes for ab initio total-energy calculations using a plane-wave basis set, Phys. Rev. B 54 (1996) 11169.

19. J.P. Perdew, K. Burke, M. Ernzerhof, Generalized Gradient Approximation Made Simple, Phys. Rev. Lett. 77 (1996) 3865.

20. P.E. Blochl, Projector augmented-wave method, Phys. Rev. B 50 (1994) 17953.

21. S. Nosé, A unified formulation of the constant temperature molecular dynamics method. J. Chem. Phys. 81 (1) (1984) 511-519.

22. S. Nosé, Constant Temperature Molecular Dynamics Methods. Prog. Theor. Phys. Suppl. 103 (1991) 1-46.

23. B. Boates, S. Hamel, E. Schwegler, S.A. Bonev, Structural and optical properties of liquid $\mathrm{CO}_{2}$ for pressures up to $1 \mathrm{TPa}$, The Journal of Chemical Physics 134 (2011) 064504.

24. B. Boates, A.M. Teweldeberhan, S.A. Bonev, Stability of dense liquid carbon dioxide, PNAS 109 (37) (2012) 14808-14812.

25. F.J. Lovas, E. Tiemann, J.S. Coursey, S.A. Kotochigova, J. Chang, K. Olsen, R.A. Dragoset, NIST Diatomic Spectral Database, (2002) http://www.nist.gov/pml/data/msddi/index.cfm.

26. G. Herzberg, Electronic spectra and electronic structure of polyatomic molecules. Van Nostrand, New York, 1966.

27. K. Kuchitsu, (ed) Structure of Free Polyatomic Molecules - Basic Data, Springer-Verlag, Berlin, 1998.

28. B. Boates, S.A. Bonev First-order liquid-liquid phase transition in compressed nitrogen, Phys. Rev. Lett. 102 (2009) 015701.

29. M.A. Morales, C. Pierleoni, E. Schwegler, D.M. Ceperley, Evidence for a first-order 
liquid-liquid transition in high pressure hydrogen from ab initio simulations, PNAS, 107 (29) (2010) 12799-12803.

30. K.P. Huber, G. Herzberg, Molecular Spectra and Molecular Structure. IV. Constants of Diatomic Molecules, Van Nostrand Reinhold Co., New York, 1979.

31. B.L. Sherman, H.F. Wilson, D. Weeraratne, B. Militzer, Ab Inition Simulations of Hot, Dense Methane During Shock Experiments, Physical Review B 86 (2012) 224113.

32. R. Chau, S. Hamel, and W.J. Nellis, Chemical processes in the deep interior of Uranus, Nature Communications 2 (2011) 203.

\section{Figure Captions and Tables}

Fig. 1. Preliminary phase diagram of $\mathrm{CO}$ based on phases observed in performed simulations. The green dasheddotted line represents the transition between majority molecular carbon and majority 2-coordinated (polymeric) carbon. Squares mark melting points determined through single-phase isothermal compression simulations, the dashed line thus represents a lower bound of the true melting line. The red solid line marks an isentrope calculated for Uranus' ice layer [14]. Results from simulations marked as A and B are shown in Fig. 2 and Fig. 3.

Fig. 2. Molecular dynamics snapshots showing three observed structures of CO: (A) molecular liquid t $2500 \mathrm{~K}$ and $0.82 \mathrm{~g} / \mathrm{cc}(2.30 \mathrm{GPa}),(\mathbf{B})$ polymeric liquid at $3500 \mathrm{~K}$ and $2.00 \mathrm{~g} / \mathrm{cc}(13.63 \mathrm{GPa})$, and $(\mathbf{C})$ partially dissociated molecular liquid at $9000 \mathrm{~K}$ and $0.82 \mathrm{~g} / \mathrm{cc}(4.37 \mathrm{GPa})$. The corresponding pair correlation functions are shown in Fig. 3 . Carbon and oxygen atoms are depicted by the green and white spheres respectively. The blue isosurface shows the density of the valence electrons.

Fig. 3. Pair correlation functions for simulations showing three different structures. (A) molecular liquid, (B) polymeric liquid, (C) partially dissociated liquid. Here A, B, and C refer to the same simulations whose snapshots are shown in Fig. 2 and plotted in Fig. 1.

Fig. 4. P-V equation of state along three isotherms plotted along with calculated and experimentally derived points on the $\mathrm{CO}$ shock Hugoniot curve [13]. The decrease in slope of the P-V curve $\leq 3500 \mathrm{~K}$ is more distinct when plotted as a $\mathrm{P} * \mathrm{~V}$ v. V curve. Only the two lowest pressure experimental data points correlate to temperatures sampled in this study. 
Leonhardi and Militzer, Liquid CO at High-P

Table 1. Pressure and internal energy from our FPMD simulations along three isotherms. Parentheses denote the $1 \sigma$ error bars.

\begin{tabular}{|c|c|c|c|}
\hline $\mathrm{T}(\mathrm{K})$ & $\rho(\mathrm{g} / \mathrm{cc})$ & $\mathrm{P}(\mathrm{GPa})$ & $\mathrm{E}(\mathrm{eV} / \mathrm{CO})$ \\
\hline $2500^{A}$ & 0.823 & $2.30(2)$ & $-0.51482(7)$ \\
\hline 3500 & 0.823 & $2.73(2)$ & $-0.50049(8)$ \\
\hline 5000 & 0.823 & $3.61(3)$ & $-0.4825(2)$ \\
\hline $9000^{C}$ & 0.823 & $4.37(6)$ & $-0.391(4)$ \\
\hline 2500 & 0.954 & $2.99(2)$ & $-0.51061(8)$ \\
\hline 3500 & 0.954 & $3.82(3)$ & $-0.4990(2)$ \\
\hline 5000 & 0.954 & $4.77(2)$ & $-0.4788(3)$ \\
\hline 2500 & 1.106 & $4.28(3)$ & $-0.50783(9)$ \\
\hline 3500 & 1.106 & $5.09(3)$ & $-0.4963(4)$ \\
\hline 5000 & 1.106 & $5.96(5)$ & $-0.4734(4)$ \\
\hline 2500 & 1.283 & $6.06(4)$ & $-0.5032(1)$ \\
\hline 3500 & 1.283 & $6.98(6)$ & $-0.4908(2)$ \\
\hline 5000 & 1.283 & 7.96(7) & $-0.4674(4)$ \\
\hline 2500 & 1.487 & $8.927(87)$ & $-0.5066(3)$ \\
\hline 3500 & 1.487 & $10.02(9)$ & $-0.4865(2)$ \\
\hline 5000 & 1.487 & $10.56(10)$ & $-0.4652(6)$ \\
\hline 2500 & 1.725 & $10.54(6)$ & $-0.5084(3)$ \\
\hline 3500 & 1.725 & $12.62(9)$ & $-0.4853(4)$ \\
\hline 5000 & 1.725 & 13.19(13) & $-0.4594(5)$ \\
\hline 2500 & 2.000 & $11.74(10)$ & $-0.5137(8)$ \\
\hline $3500^{\mathrm{B}}$ & 2.000 & $13.63(8)$ & $-0.4982(6)$ \\
\hline 5000 & 2.000 & $17.34(17)$ & $-0.4540(6)$ \\
\hline 2500 & 2.319 & $17.74(17)$ & $-0.5081(5)$ \\
\hline 3500 & 2.319 & $20.74(19)$ & $-0.4891(3)$ \\
\hline 5000 & 2.319 & $24.30(24)$ & $-0.4427(6)$ \\
\hline 3500 & 2.689 & $30.45(22)$ & $-0.4746(7)$ \\
\hline 5000 & 2.689 & $35.70(23)$ & $-0.4332(7)$ \\
\hline 3500 & 3.118 & $45.59(31)$ & $-0.4644(6)$ \\
\hline 5000 & 3.118 & $52.31(37)$ & $-0.4181(5)$ \\
\hline 5000 & 3.616 & $82.03(43)$ & $-0.4000(8)$ \\
\hline 5000 & 4.193 & $136.11(46)$ & $-0.3742(6)$ \\
\hline
\end{tabular}

${ }_{\mathrm{A}, \mathrm{B}, \mathrm{C}}$ Pair correlation functions and snapshots from these simulations are provided in Fig. 3 and Fig. 2 respectively. 
Figure 1

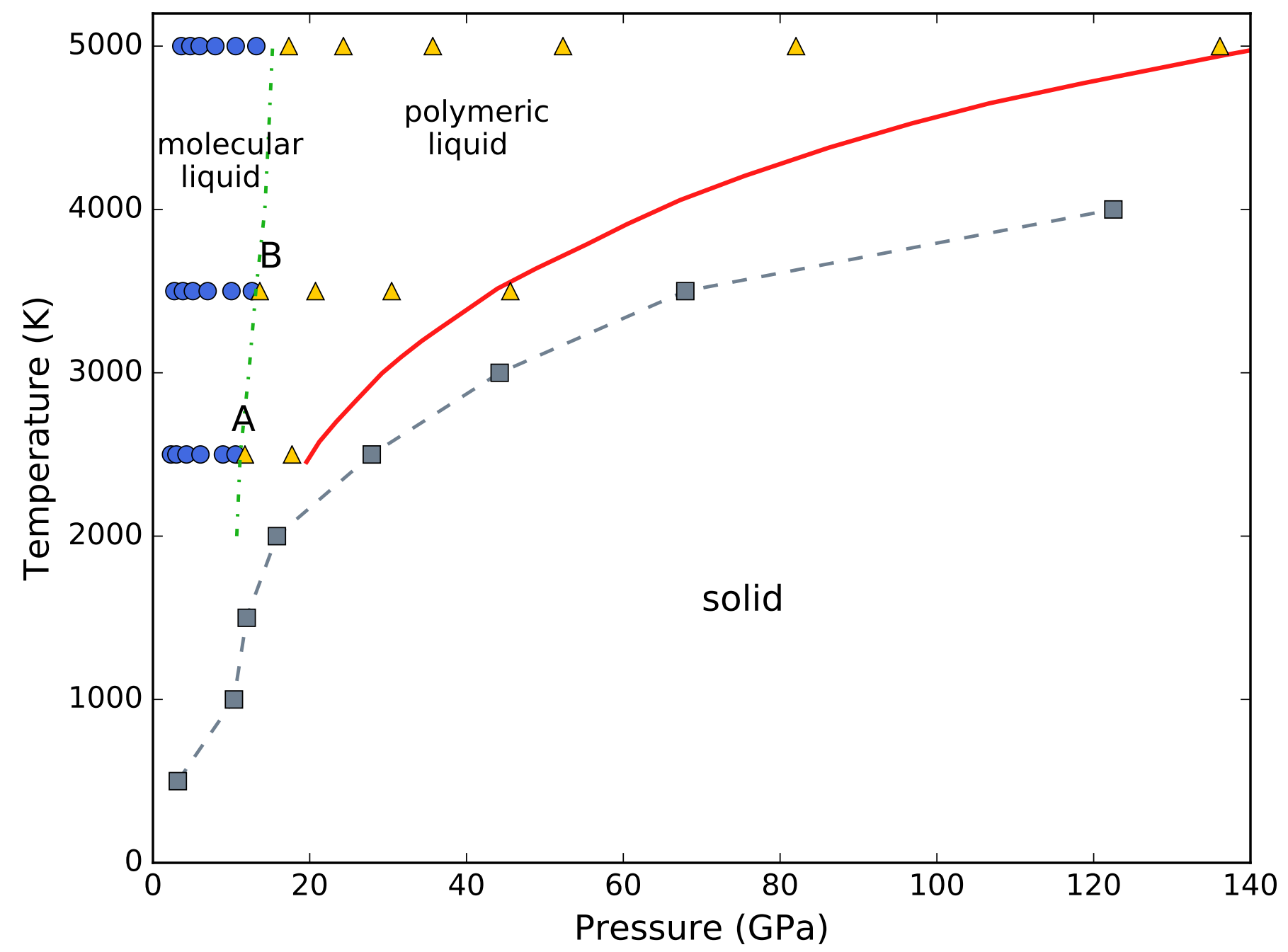




\section{Figure 2}

A

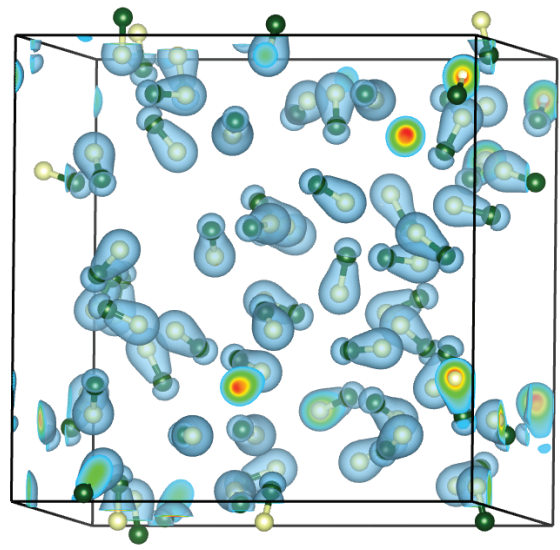

B

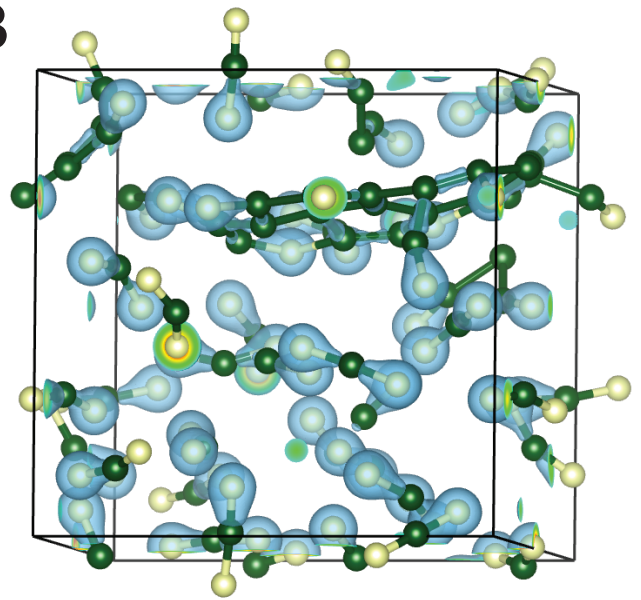

C

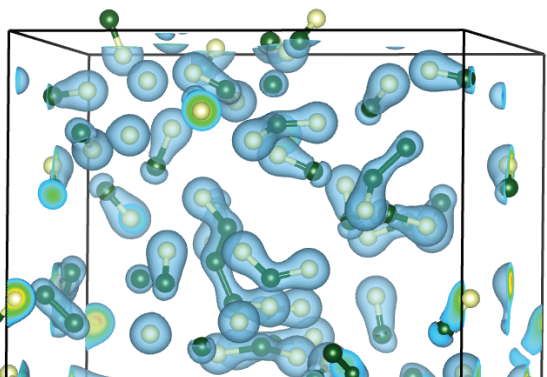

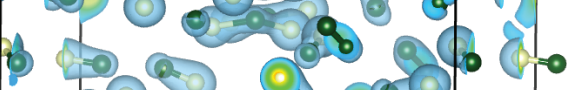

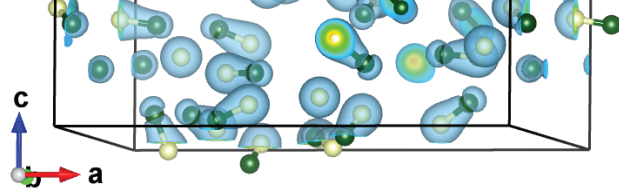




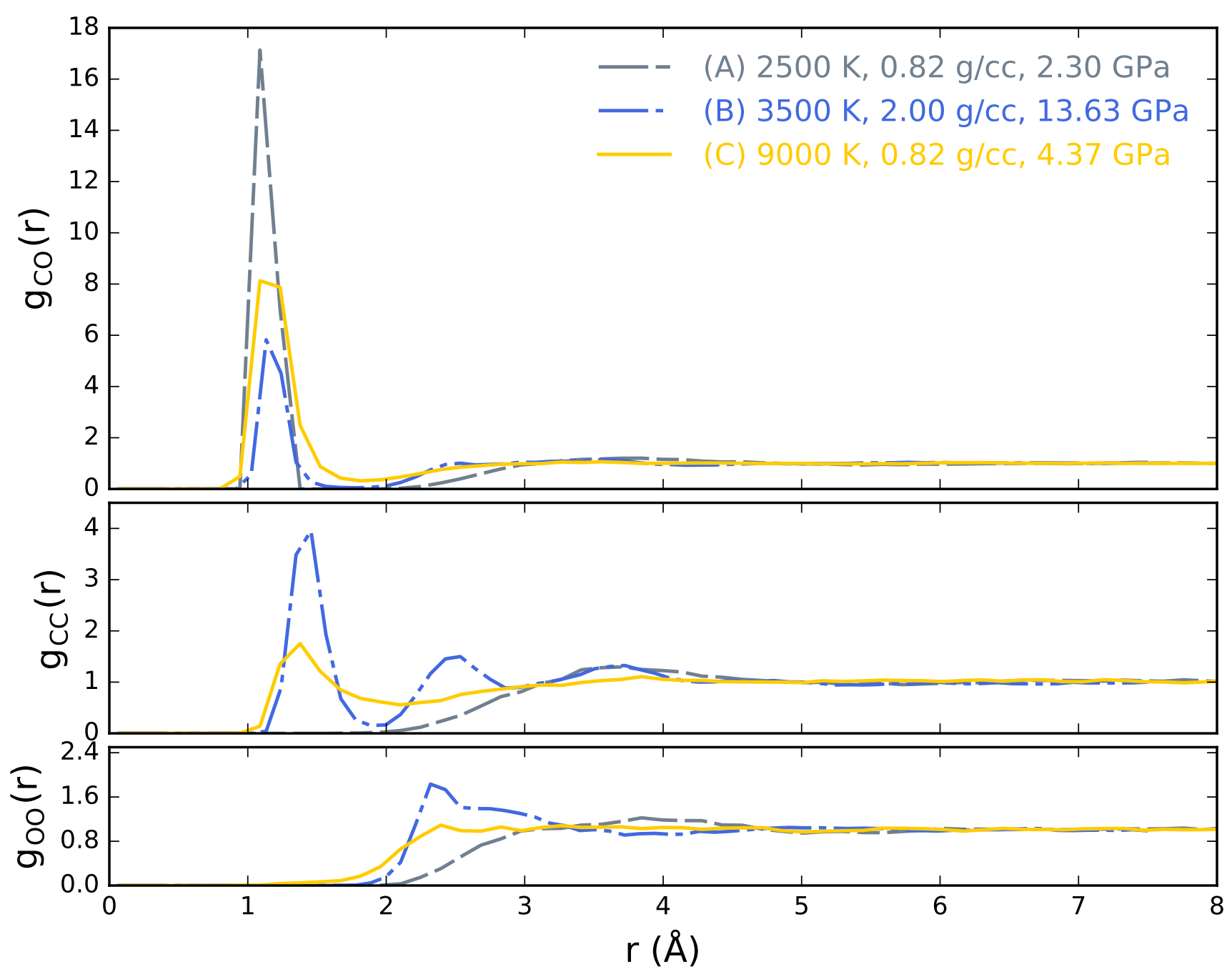


Figure 4
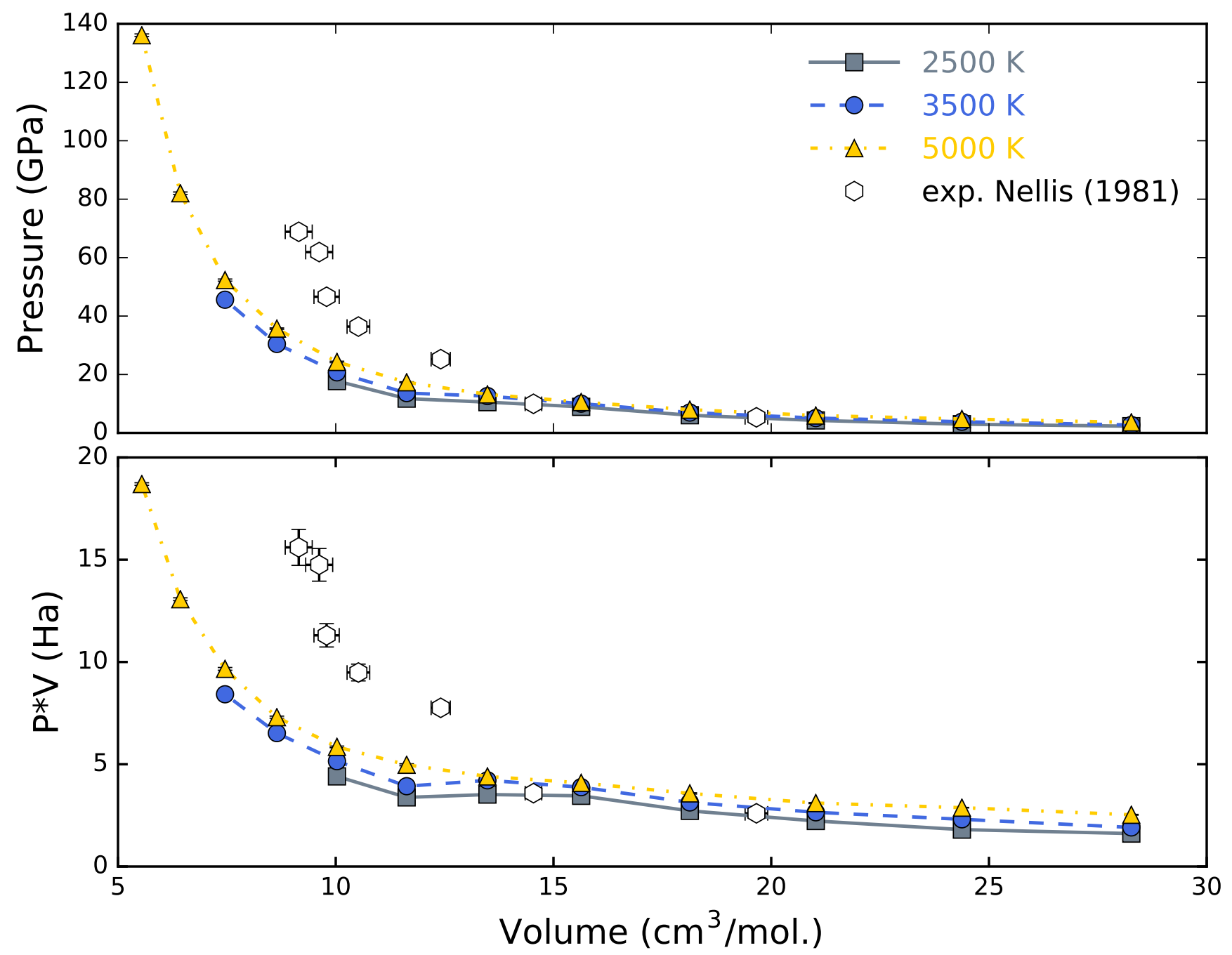